\title{
Performance and operation experience of the ATLAS Semiconductor Tracker
}

\author{
Paul John Dervan*i \\ University of Liverpool, UK \\ E-mail: Paul.Dervan@cern.ch \\ Taka Kondo \\ KEK, Japan \\ E-mail: kondo@post.kek.jp
}

\begin{abstract}
After more than 3 years of successful operation at the LHC, we report on the operation and performance of the ATLAS Semi-Conductor Tracker (SCT) functioning in a high luminosity, high radiation environment. The SCT is constructed of 4088 silicon detector modules, for a total of 6.3 million strips. Each module is designed, constructed and tested to operate as a stand-alone unit, mechanically, electrically, optically and thermally. The modules are mounted into two types of structures: one barrel (4 cylinders) and two end-cap systems ( 9 disks on each end of the barrel). The SCT silicon micro-strip sensors are processed in the planar p-in-n technology. The signals are processed in the front-end ABCD3TA ASICs, which use a binary readout architecture. Data is transferred to the off-detector readout electronics via optical fibres. We find $99.3 \%$ of the SCT modules are operational and the hit efficiency exceeds the design specifications. We will report on the operation and performance of the detector, including an overview of the issues encountered and report on the expected observation of a significant increase in leakage currents from bulk damage due to non-ionizing radiation and make comparisons with the predictions. We will also cover the time evolution of the key parameters of the strip tracker, including the evolution of noise and gain in the harsh LHC environment.
\end{abstract}

11th International Conference on Large Scale Applications and Radiation Hardness of Semiconductor Detectors

3-5 July 2013

Auditorium Cassa di Risparmio di Firenze, via Folco Portinari 5, Florence, Italy

* Speaker.

†n behalf of the ATLAS SCT Collaboration 


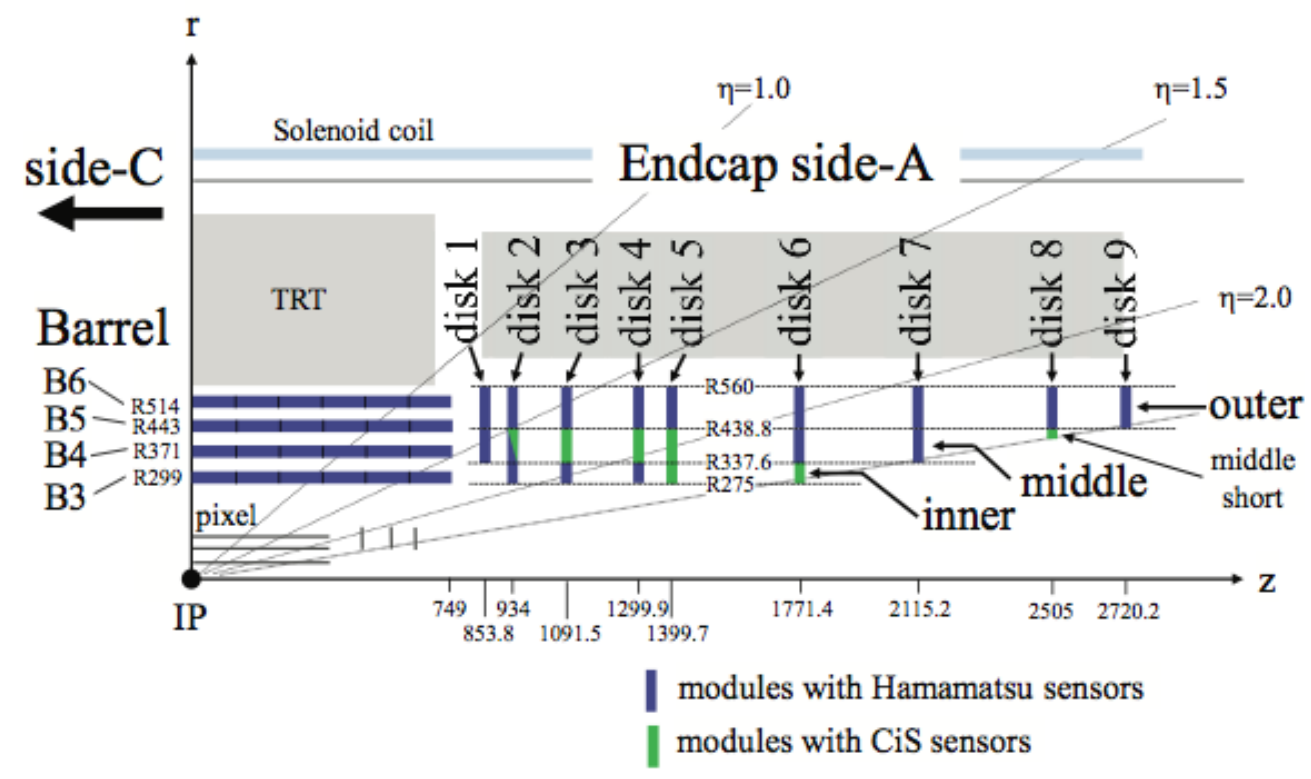

Figure 1: A quarter section of the inner tracker of ATLAS.

\section{Introduction}

The SCT is part of the inner tracker of the ATLAS [1] experiment at the Large Hadron Collider [2]. The ATLAS experiment consists of the inner tracker surrounded by the calorimetry and muon chambers. The inner tracker, shown in Figure 1, consists of three sub-detectors. The nearest to the beam line, and moving out in radius, is the silicon pixel detector, then a silicon strip detector (SCT) and finally a straw tube tracker, the Transition Radiation Tracker (TRT). This paper will describe the performance of the SCT over its first three years of operation.

\section{Semi-Conductor Tracker}

The SCT consists of $61 \mathrm{~m}^{2}$ of active silicon-strip detector modules with 6.3 million readout channels. It is made up of 4088 silicon modules in four barrels and eighteen end-cap disks (nine on each side of the barrel, called end-caps $\mathrm{A}$ and $\mathrm{C}$ ). The silicon modules are operated at $-7^{\circ} \mathrm{C}$ to $+6^{\circ} \mathrm{C}$, achieved with an evaporative cooling system using $\mathrm{C}_{3} \mathrm{~F}_{8}$ [3]. The layout has been designed so that the SCT covers a radial region from $30 \mathrm{~cm}$ to $52 \mathrm{~cm}$ and has an azimuthal coverage out to $|\eta|$ $=2.5$. This ensures that energetic particles pass through at least four layers.

\subsection{SCT sensors}

All SCT sensors are single-sided p-in-n, $285 \mu \mathrm{m}$ thick, and have $768+2$ AC-coupled strips. The endcaps and barrels comprise 6944 and 8448 sensors respectively. 367 of the barrel sensors are $\langle 100\rangle$, but all other sensors are $<111>$. The barrel are $60.0 \times 63.6 \mathrm{~mm}^{2}$ with an $80 \mu \mathrm{m}$ strip 
Table 1: The number of defective channels in the SCT (as of Feburary 2013).

\begin{tabular}{|c|c|c|c|c|c|}
\hline \multirow{2}{*}{ Components } & \multirow{2}{*}{ Total } & \multicolumn{4}{|c}{ Defect Components } \\
& & Barrel & Endcap & SCT & Fraction (\%) \\
\hline Modules & 4088 & 11 & 19 & 30 & $0.73 \%$ \\
\hline Readout ASICs & 49056 & 38 & 11 & 49 & $0.10 \%$ \\
\hline Strips & 6279168 & 4111 & 8020 & 12131 & $0.21 \%$ \\
\hline
\end{tabular}

pitch. All of them were manufactured by Hamamatsu. The end-cap is made up of wedge sensors with five different shapes. The strip pitch varies from $56.9-90.4 \mu \mathrm{m}$. $74.9 \%$ were manufactured by Hamamatsu, $17.1 \%$ by CIS and the remaining $8 \%$ were CIS oxygen enriched sensors (for the inner radius of the disks). The nominal operational bias voltage is $150 \mathrm{~V}$, and the typical depletion voltage before irradiation was approximately $80 \mathrm{~V}$. The location of the various sensor types is shown in Figure 1.

\subsection{SCT Modules}

There are 2112 modules in the barrel and 988 in each end-cap (called A and C). The modules come in several shapes and sizes. The barrel modules [4] are rectangular, with the hybrids (holding the ABCD3TA readout ASICs [5]), spanning the centre. The end-cap modules [6] come in four different wedge shapes. Starting at the smallest radius and working out: there are inner, middle, short middle and outer modules, and these have the hybrid attached at one end. All the modules are made up of four silicon sensors, two sensors on each side of the module. except for the endcap short middle and inner modules. These consist of only two sensors, one on each side.

The modules are constructed on a thermally conductive base plate, which in addition to providing mechanical support, conducts heat away from the module to the cooling-blocks. The two layers of sensors are rotated with respect to one another by 40 milliradian, in order to get a 3D point. The data to and from the modules are transmitted on fibre optics to the off-detector electronics.

\section{Performance}

\subsection{Operational Efficiency}

The SCT was installed into ATLAS, and was ready for the first LHC proton-proton collisions at a centre of mass energy of $7 \mathrm{GeV}$ in March 2010. Since then and until the end of running in February 2013, more than $99 \%$ of the 6.3 million readout channels were operational. Details of the modules, readout ASICs and sensor strips are shown in Table 1. Thirteen of the defective end-cap modules are due to a cooling pipe failure.

\subsection{Cooling and module temperatures}

Cooling of the modules is provided by a $\mathrm{C}_{3} \mathrm{~F}_{8}$ evaporative cooling system. The hybrid temperature of the modules is shown in Figures 2 and 3 for the barrel and end-cap. For Barrel 3, the innermost SCT layer, it can be seen that there is one cooling circuit that has a higher value, and that Barrel 6, the outermost SCT layer, is operated at a higher temperature. Barrel 6 is operated at 

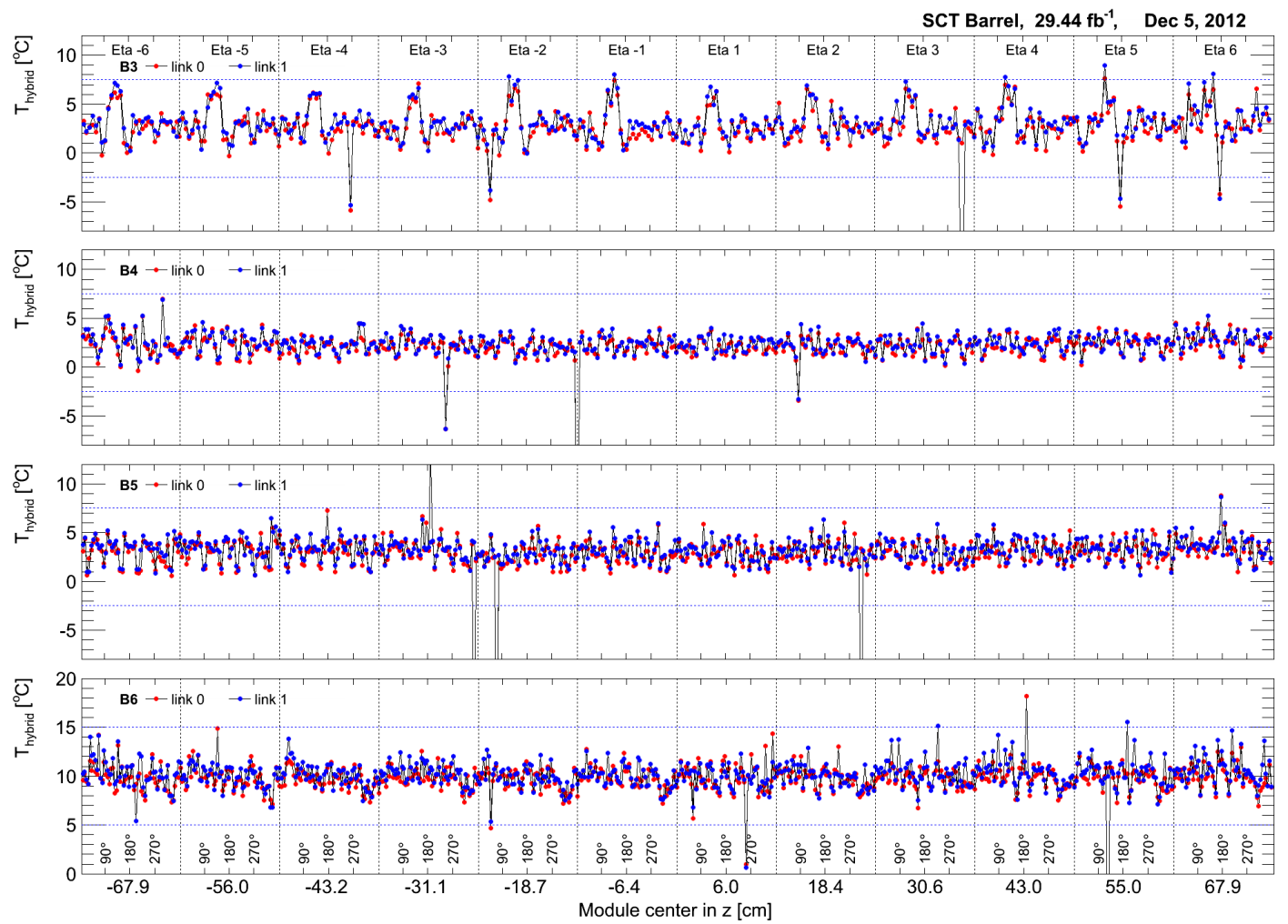

Figure 2: Barrel modules hybrid temperature. Barrel 3 is shown at the top and barrel 6 is the bottom plot.

a higher temperature to act as a thermal shield between the SCT and the TRT which has to operate at room temperature. All the cooling loops are operational except for one in the end-cap. The time history for the cooling is shown in Figure 4. It can be seen that the cooling has performed well over the three years of running. The slight increase in the barrel temperature in 2012 was due to a slight adjustment on the back pressure regulators.

For monitoring the radiation damage, we need to know the silicon temperature, rather than the hybrid temperature. The barrel silicon is known to be $3^{\circ} \mathrm{C}$ cooler than the hybrid temperature [10]. The end-cap sensors are known to be $15.5^{\circ} \mathrm{C}$ cooler for outer and middle modules and $13.1^{\circ} \mathrm{C}$ cooler for the inner modules [6].

\subsection{Radiation Damage}

The radiation damage in the SCT can be monitored using the leakage currents of the modules. The leakage current should increase with radiation damage as given by Equation 3.1 [9]. Where $I$ is the leakage current, $\phi$ the fluence (in units of $1 \mathrm{MeV} n_{e q} / \mathrm{cm}^{2}$ ), $\alpha$ the damage factor (sensitive to annealing temperature $T$ and time $t$ ) and $V$ is the active volume of the sensor.

$$
\frac{\Delta I}{\Delta \phi}=\alpha(t, T) V
$$




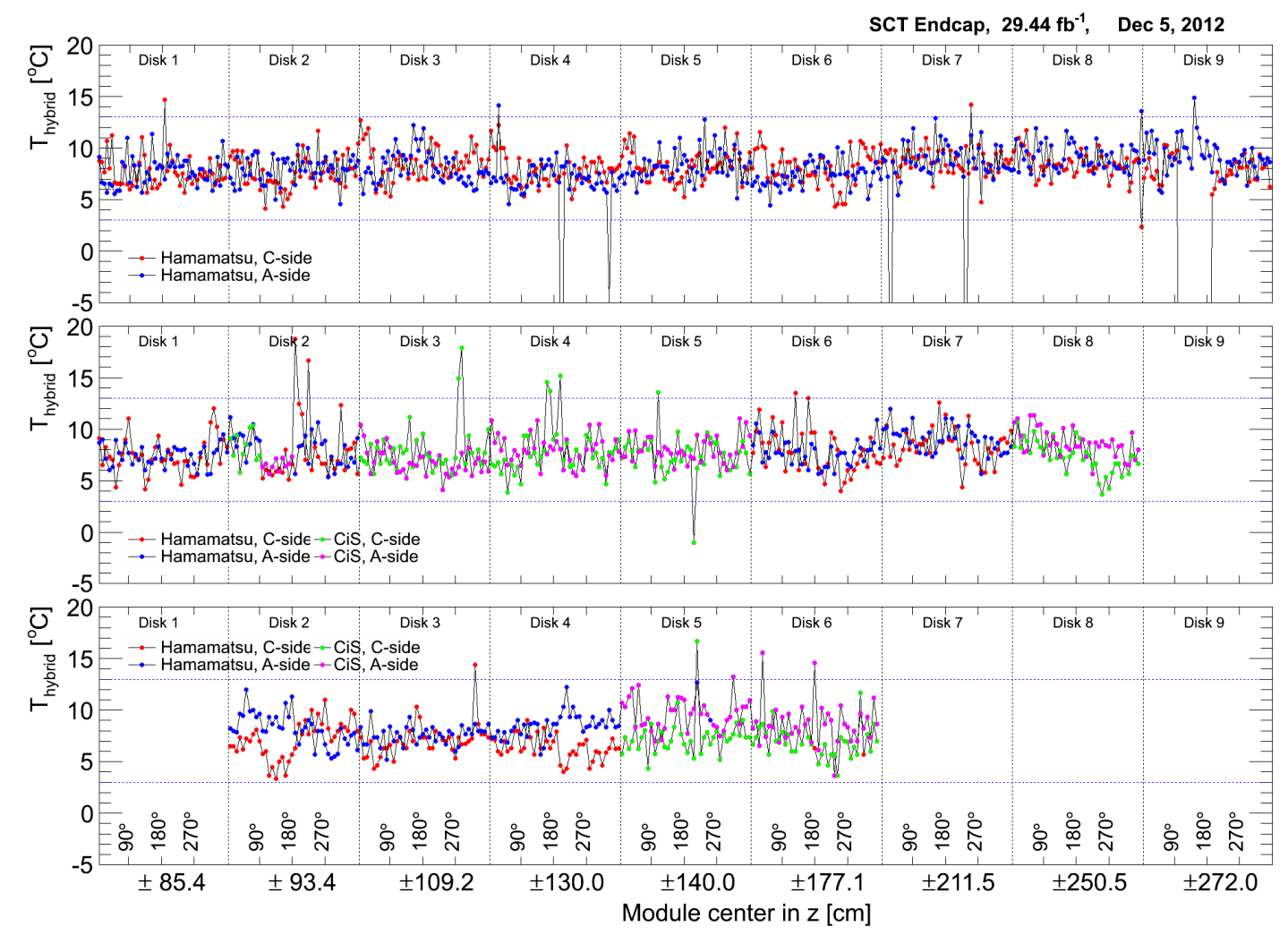

Figure 3: End-cap modules hybrid temperature. The top plot is the outer modules, the middle plot is the middle and short middle modules and the lower plot is for the inner modules.

Figure 5 shows the leakage current distribution of the SCT modules of the four barrel layers (B3 to B6) on Dec 5, 2012 when the LHC beam was off. Out of 2112 barrel modules, 2060 modules $(97.5 \%)$ satisfy the cuts of the bias voltage $=150+5-2 \mathrm{~V}$ with reasonable hybrid temperatures. The currents measured by the high voltage power supplies are converted to those at a temperature of $0^{\circ} \mathrm{C}$ using the temperature scaling formula 3.2. Where, REF is the value at $0^{\circ} \mathrm{C}$, Meas are the measured values, $\mathrm{I}$ is the current, $\mathrm{T}$ is the temperature in Kelvin, $k_{b}$ is the Bolzmann's constant and $\mathrm{E}_{\text {gen }}=1.21 \mathrm{eV}$ [11]. The leakage currents are plotted in 12 groups, each of which contains all the modules placed at the same $\mathrm{z}$ (beam direction) position. The leakage current of modules in each group are plotted in series starting from 0 to 360 degree in $\phi$ direction. The distribution of currents is very flat along $\mathrm{z}(\eta)$ except for B3 in which the central part $(|\mathrm{z}|<30 \mathrm{~cm})$ shows about $7 \%$ excess. This flat distribution is a good reflection of the flat eta distribution of secondary particles around the centre of the minimum bias events.

The depletion voltage is not expected to have changed significantly and methods of measuring it are under investigation.

$$
\frac{I_{R E F}}{I_{\text {Meas }}}=\left(\frac{T_{R E F}}{T_{\text {Meas }}}\right)^{2} \exp \left[-\frac{E_{\text {gen }}}{2 k_{B}}\left(\frac{1}{T_{R E F}}-\frac{1}{T_{\text {Meas }}}\right)\right]
$$

In Figure 6, the leakage currents measured by the HV power supply of the ATLAS SCT barrel 


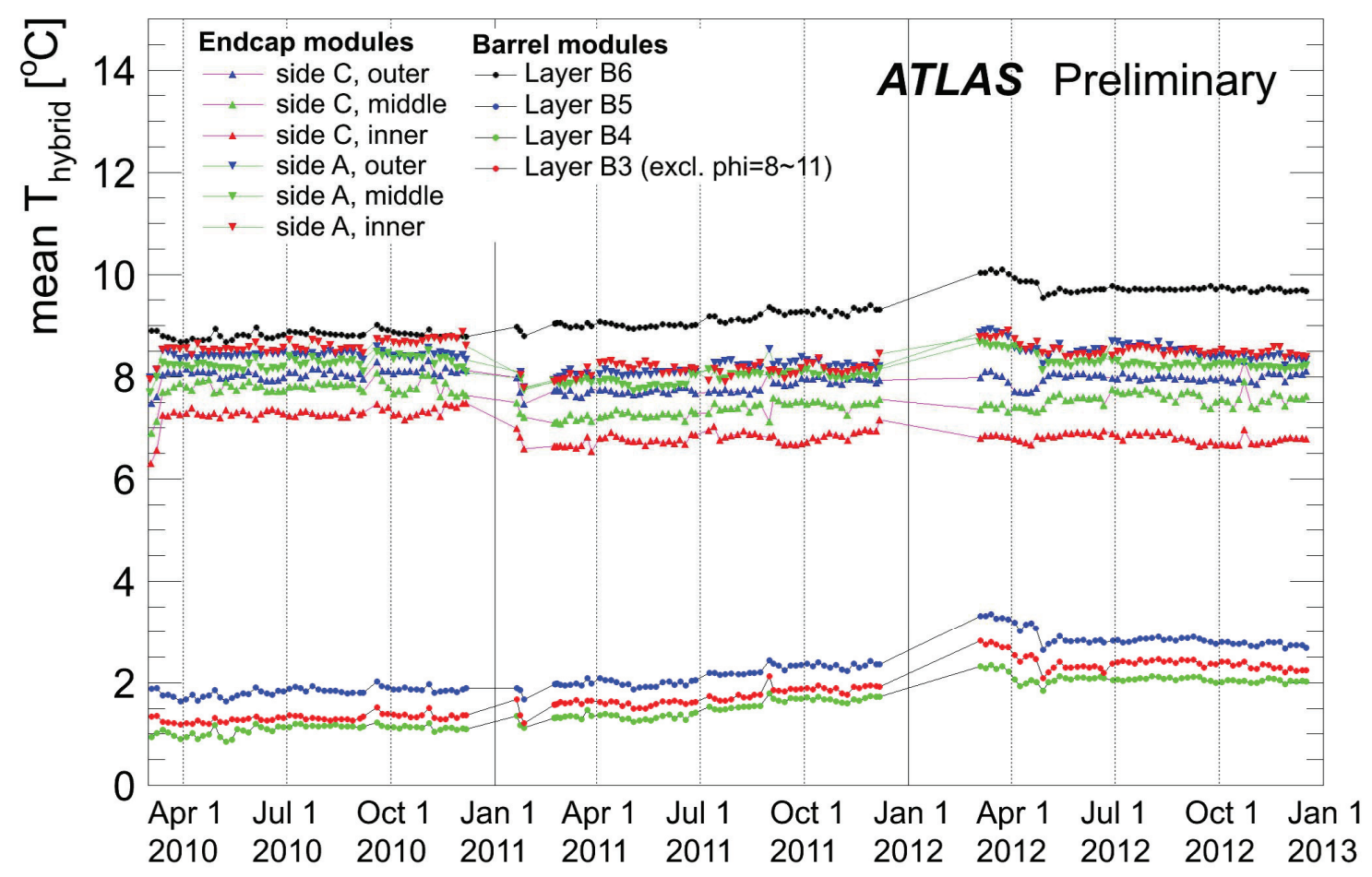

Figure 4: The time history of the hybrid temperatures (averaged over 5 days).

modules are shown for the four SCT barrel layers (B3 to B6). The data was taken when the LHC beams were off. The predicted leakage currents by the Hamburg /Dortmund model [12, 13] are shown by four lines with colored bands indicating 1 sigma uncertainties, which is obtained by quadratically summing up all uncertainties of the model parameters as well as the temperature measurements. The prediction takes into account the self-annealing effects using the measured sensor temperatures shown at the top of plots. The prediction is based on the total 7 and $8-\mathrm{TeV}$ collision luminosities delivered at Point-1, shown by the black solid line. Results of the FLUKA simulation [14] of minimum bias events at $7 \mathrm{TeV}$ pp collisions (with a 5\% increase at $8 \mathrm{TeV}$ ) are used to convert the collision luminosity to $1 \mathrm{MeV}$ neutron-equivalent fluence at each layer. The error of the FLUKA simulation is not included in the estimate of 1 sigma uncertainty. Very good agreement between data and predictions are observed over 4 decades in leakage current as well as over 3 years of operation, indicating (1) the observed HV currents are mostly due to bulk generation current, (2) the leakage current models with self-annealing terms are well applicable and (3) the flux simulation is reasonable. In conclusion, the leakage current is a good measure of the radiation levels in the SCT region.

Figure 7 shows the corrected leakage current for the end-caps. One can see that the EndcapA modules have a systematically higher leakage current. This is due to the temperature of the nitrogen that is flushed through the detector. Also, Disk 8 and 9 also show higher currents, which 


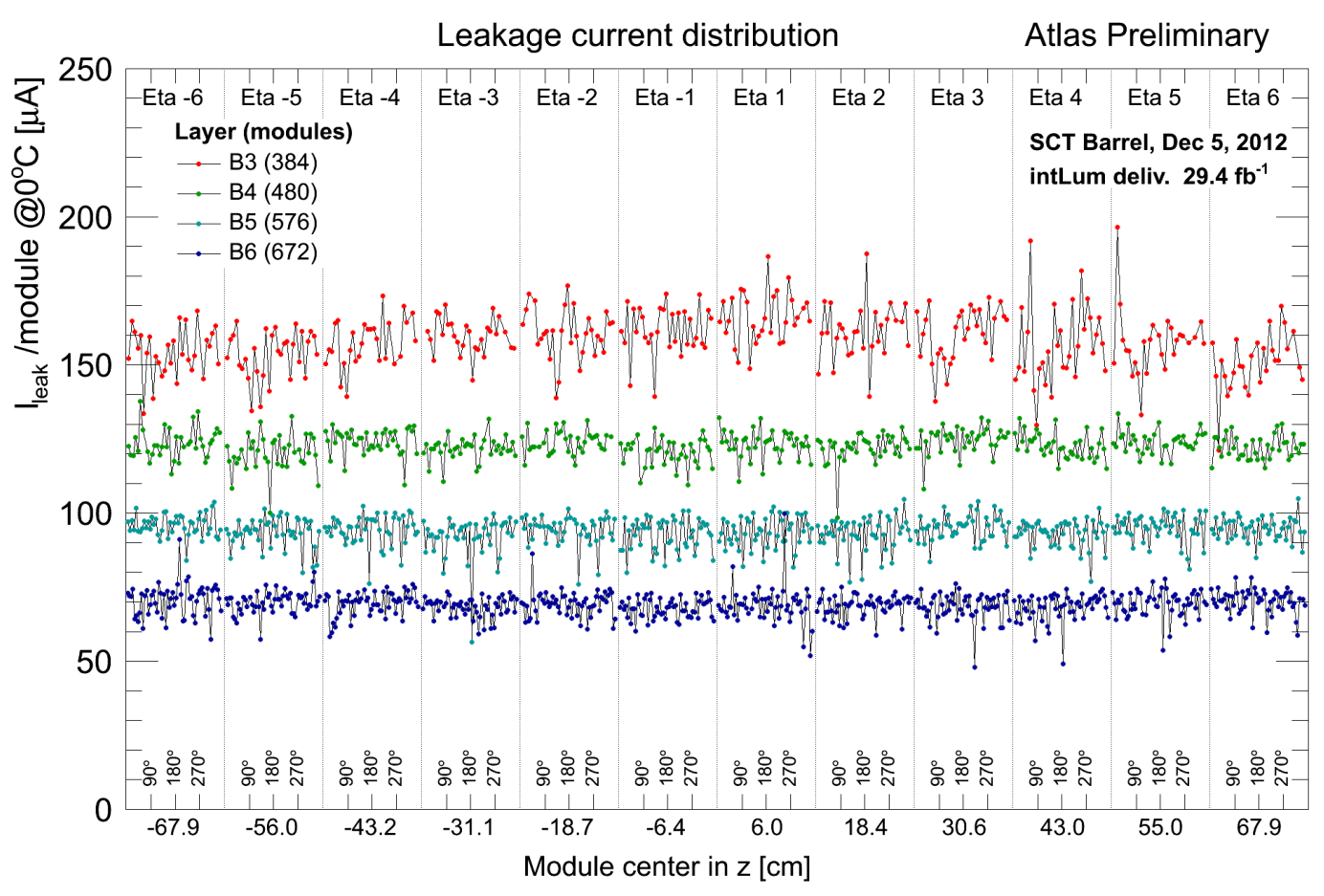

Figure 5: The corrected barrel module leakage currents.

was predicted from the FLUKA simulations, and is due to the higher radiation levels in this area. Due to the high variation in the currents and uncertainties on the module temperatures it is difficult to normalise the currents to $0^{\circ} \mathrm{C}$. This is work in progress.

\subsection{Noise}

Figure 8 shows the distributions of the ASICs averaged noise as of October 2010 (top) and December 2012 (bottom). Only modules with a bias voltage values greater than $145 \mathrm{~V}$ are selected (this is to ensure that the silicon detectors are fully depleted). For the barrels, only modules with $<111>$ sensors are plotted, while for the end-caps, ASICs with Hamamatsu and CiS sensors are shown separately. After receiving $30 \mathrm{fb}^{-1}$ of integrated luminosity (December 2012), the noise values stayed nearly at the original level for barrel and end-cap outer modules, while they increased about $10 \%$ in the end-cap short middle modules and about $5 \%$ in inner modules both with Hamamatsu and CiS sensors.

The change in the noise and gain (gain of the ABCD ASICs) of the modules over time is shown in Figure 9. It can be seen that the noise of modules built with $<111>$ silicon decreased in 2010, but not for the $<100>$ silicon. Also the $10 \%$ increase in the CIS middle modules can be seen. The gain of all the ASICs decreased in the middle of 2011 and has stayed relatively level since that time. The drop is believed to be due to surface charge effects. 


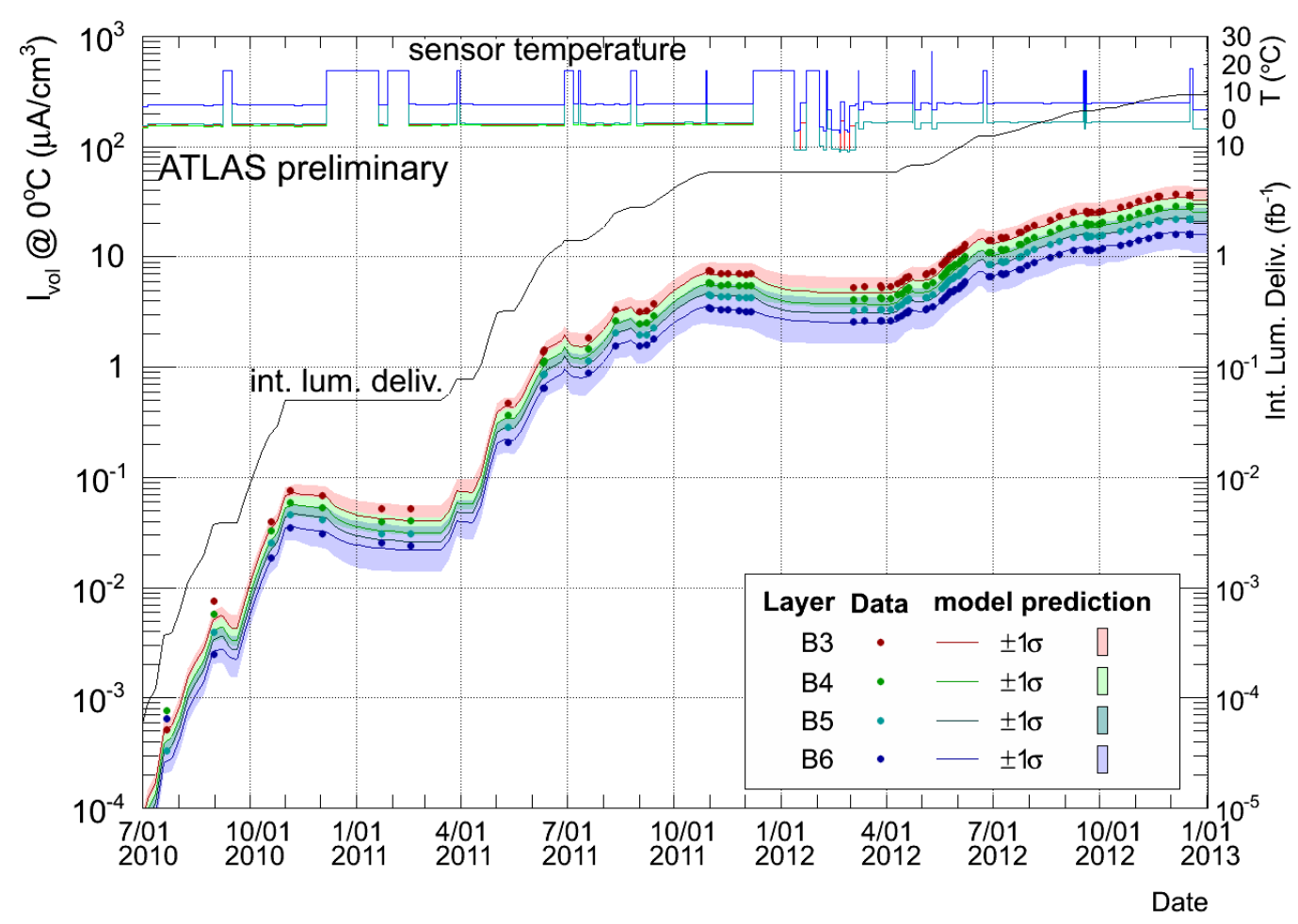

Figure 6: The corrected barrel leakage current measurements compared to the Hamburg/Dortmund radiation damage model. The top of the plot shows the average temperature of the sensors. The luminosity is shown by the solid black line.

\subsection{Hit Efficiency}

The hit efficiency for the SCT can be seen in Figure reffigure-trackin1 for low luminosity running and 11 for high luminosity running. The efficiency is calculated by taking the number of hits (on each layer) on high transverse momentum tracks divided by the number of possible hits in the data. Not functioning readout strips were removed from the analysis. The efficiency is higher than the specification of $99 \%$. Similar results are seen in the end-caps.

\section{Summary}

After three years of operation, the SCT is performing well within its design specification. More than $99 \%$ of the detector is still operational. Radiation damage has been observed and is well described by the Hamburg/Dortmund module, in the barrel region. 


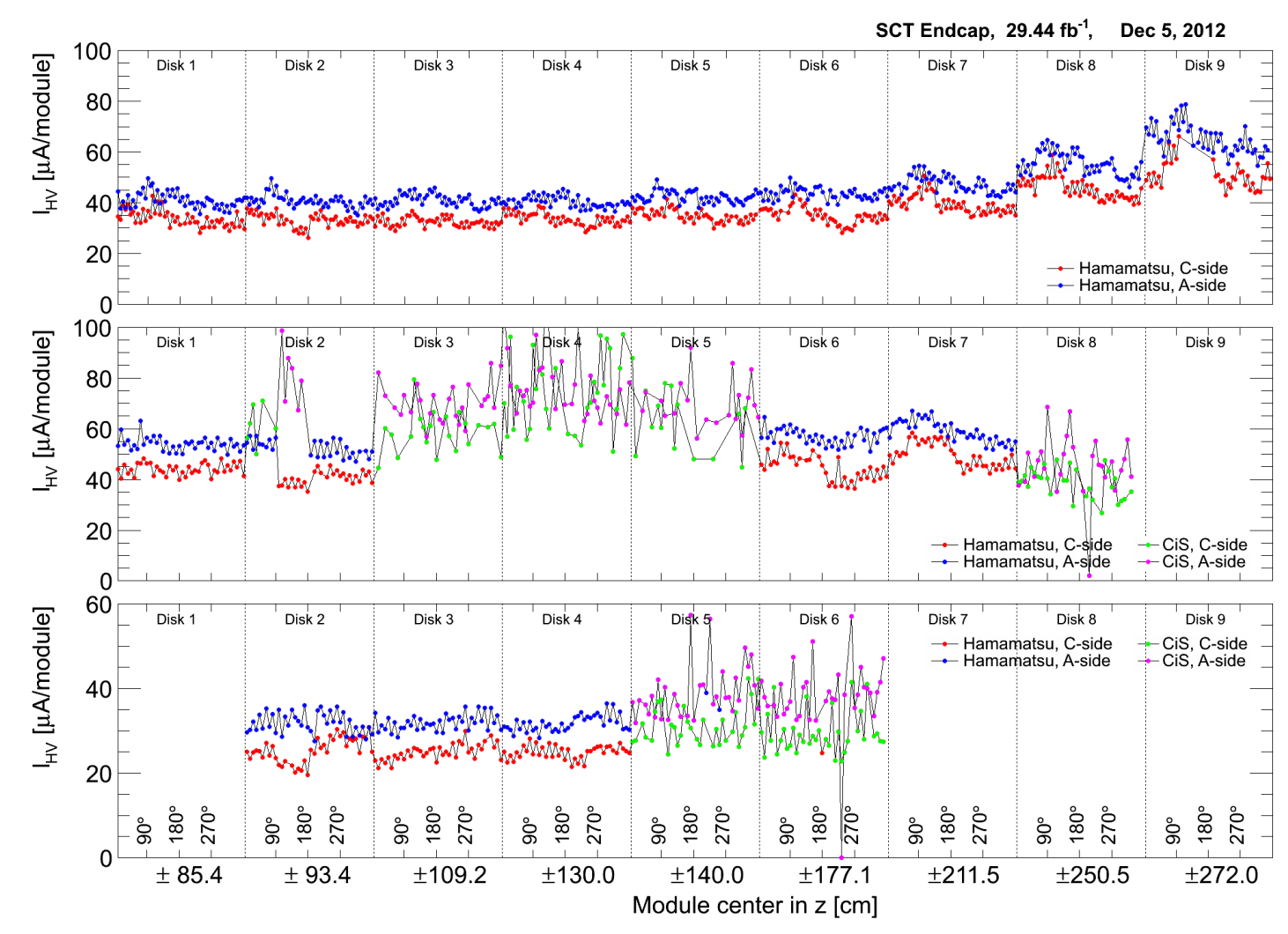

Figure 7: The corrected end-cap leakage current measurements. 


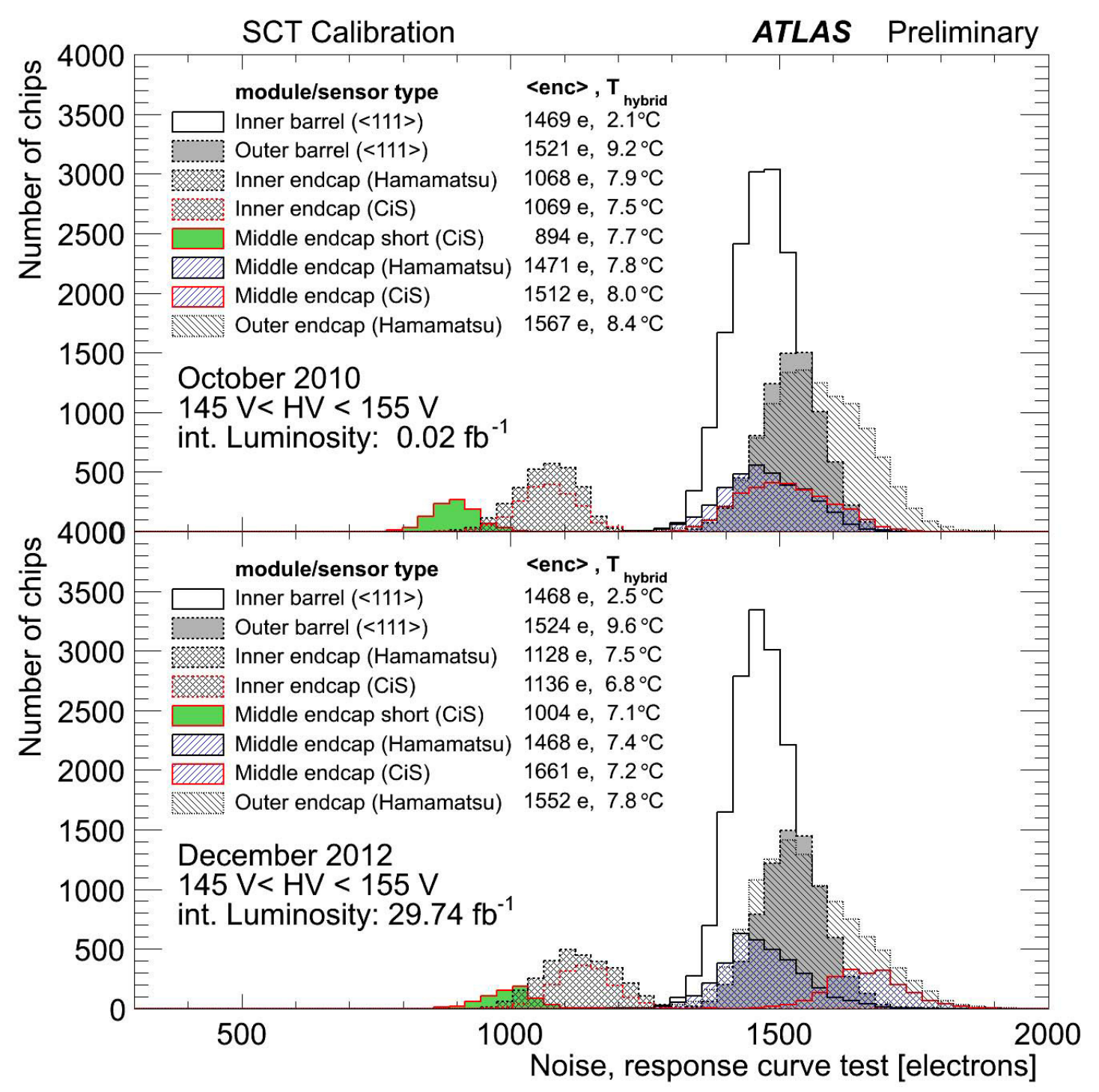

Figure 8: The noise of the various modules types at two different times. 


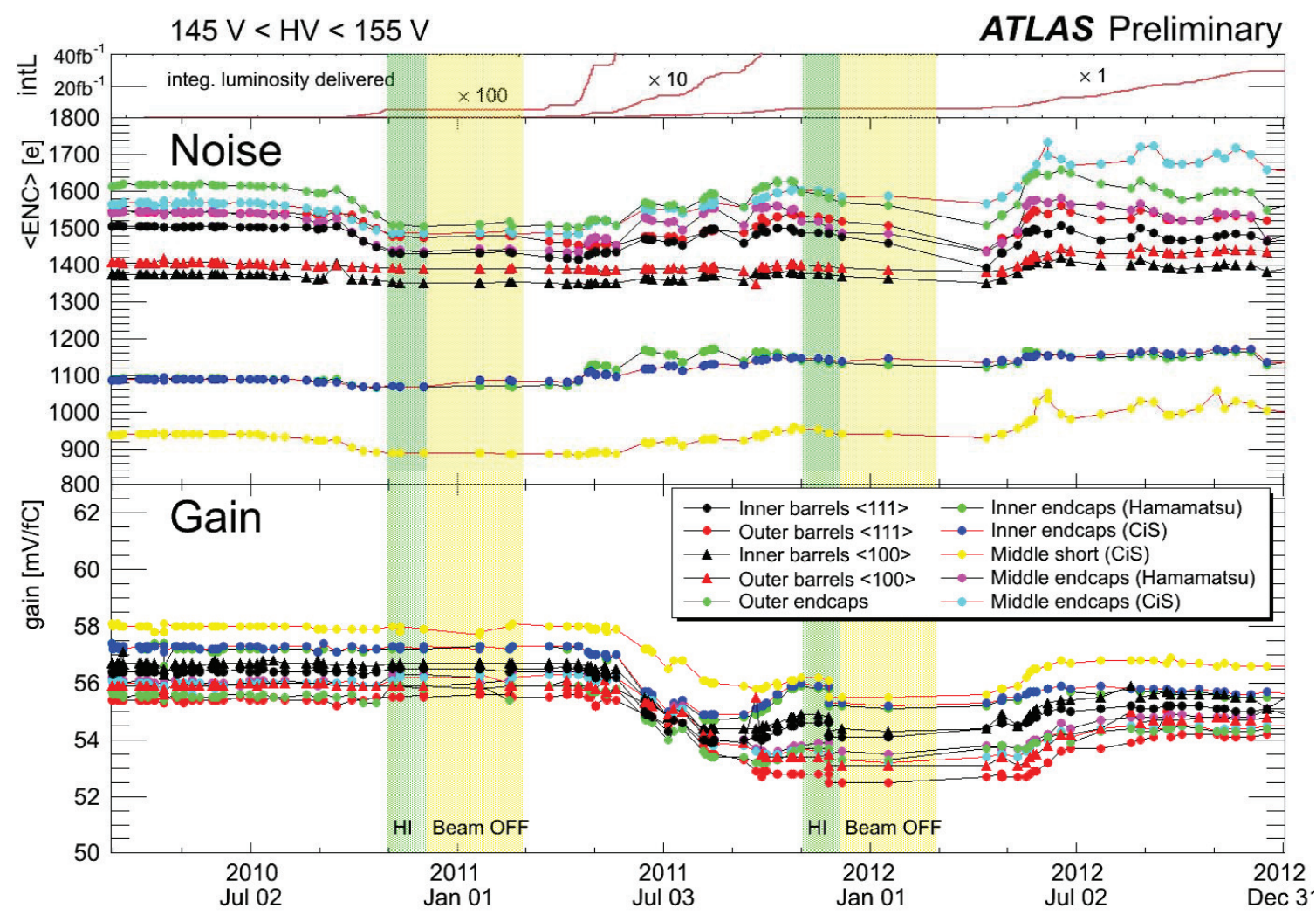

Figure 9: The change in the noise and gain of the modules over time.

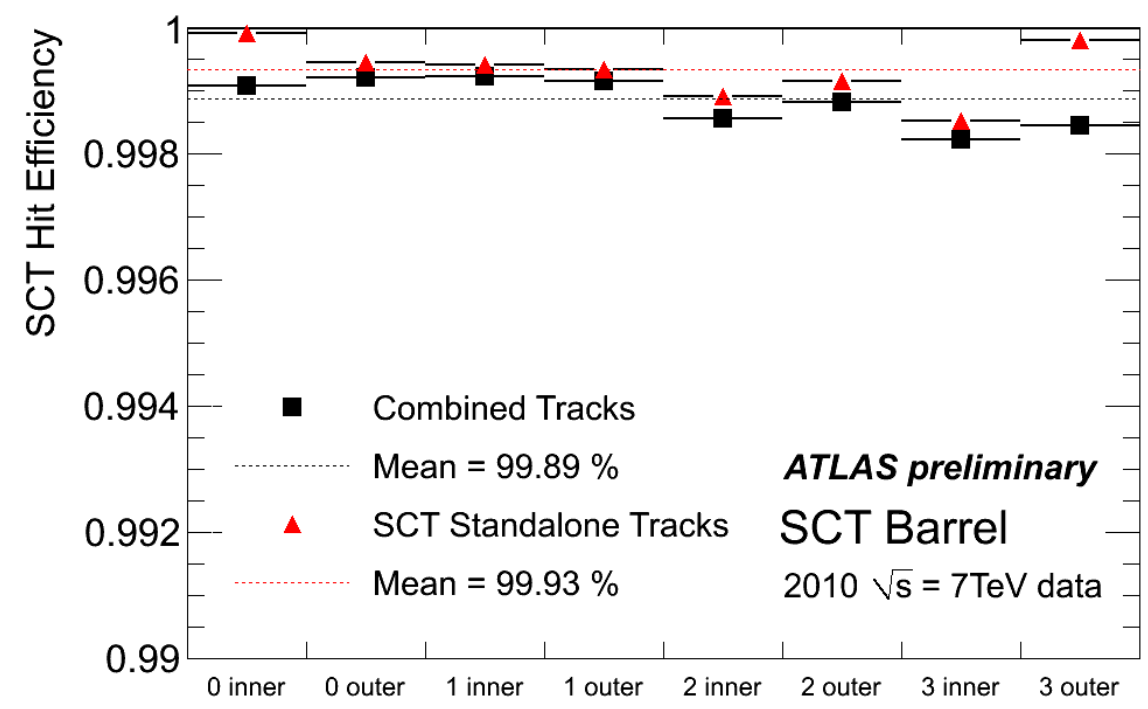

Figure 10: The SCT barrel tracking efficiency in low luminosity running (2010). This figure and all the following are taken from $[7,8]$ 


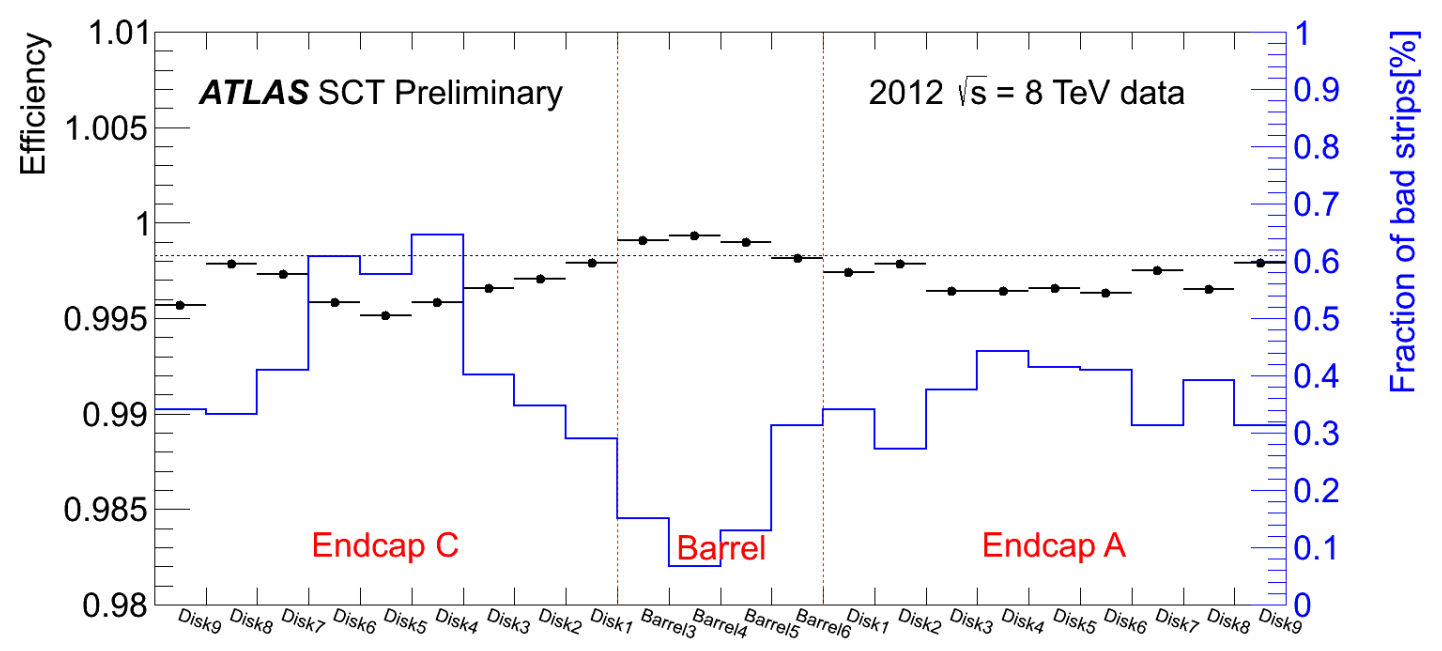

Figure 11: The SCT hit tracking efficiency (black) for high luminosity running. The blue line and right-hand axis indicates the fraction of disabled strips in each layer. 


\section{References}

[1] A. Abdesselam et al., The ATLAS experiment at the CERN Large Hadron Collider, 2008 JINST 3 S08003.

[2] L. Evans and P. Bryant, The LHC machine, 2008 JINST 3 S08001.

[3] D. Attree et al., The evaporative cooling system for the ATLAS inner detector, 2008 JINST 3 P07003.

[4] A. Abdesselam et al., The barrel modules of the ATLAS semiconductor tracker, Nucl. Instrum. Meth. A568 (2006) 642.

[5] F. Campabadal et al., Design and performance of the ABCD3TA ASIC for readout of silicon strip detectors in the ATASL semiconductor tracker, Nucl. Instrum. Meth. A552 (2005) 292.

[6] A. Abdesselam et al., The ATLAS semiconductor tracker end-cap modules, Nucl. Instrum. Meth. A575 (2007) 353 .

[7] https://twiki.cern.ch/twiki/bin/viewauth/Atlas/SctNoiseGain\#Approved_plots.

[8] https://twiki.cern.ch/twiki/bin/viewauth/Atlas/SctLeakageCurrent\#Approved_plots.

[9] The ROSE Collaboration. 2nd RD48 status report. CERN/LHCC 98-39.

[10] A. Abdesselam et al., The ATLAS barrel modules of the ATLAS semiconductor tracker, Nucl. Instrum. Meth. A568 (2006) 642.

[11] A.Chilingarov, RD50 Technical Note RD50-2011-01.

[12] M. Moll DESY-THESIS-1999-040 (Dec 1999)

[13] Oraf Krasel, Dortmund Dissertation, July 2004.

[14] https://twiki.cern.ch/twiki/bin/viewauth/Atlas/BenchmarkingAtTheLHC. 